Supporting Information for

\title{
Spin-state ice in elastically frustrated spin-crossover materials
}

\author{
Jace Cruddas* and B. J. Powell* \\ School of Mathematics and Physics, The University of Queensland, QLD 4072, Australia \\ E-mail: j.cruddas@uq.edu.au; powell@physics.uq.edu.au
}

\section{Mapping between spin ice rules and spin-state ice rules}

In the field of artificial spin ices it is traditional to name lattices with spins located on the bonds of the lattice. This is natural given the physical structure of artificial spin lattices. However, in the current work we use the locations of the metal atoms to define the vertices of the lattice. In Figure S1 we demonstrate how lattices are renamed when moving between these two nomenclatures, in particular we show that the square lattice (in the bond convention) is equivalent to the checkerboard lattice (in the site convention) and that the honeycomb (bond) lattice is equivalent to the kagome site (lattice).

Figure S1 also demonstrates that spin ice states on the bond lattices map directly onto spin-state ice rules on the site lattices after an appropriate relabeling.

\section{Snapshots of Monte Carlo simulations}

In Figure 5 of the main text we show regions of snapshots of our Monte Carlo simulations. Images of the full state of the simulations from which these are taken are shown in Figures 
a)

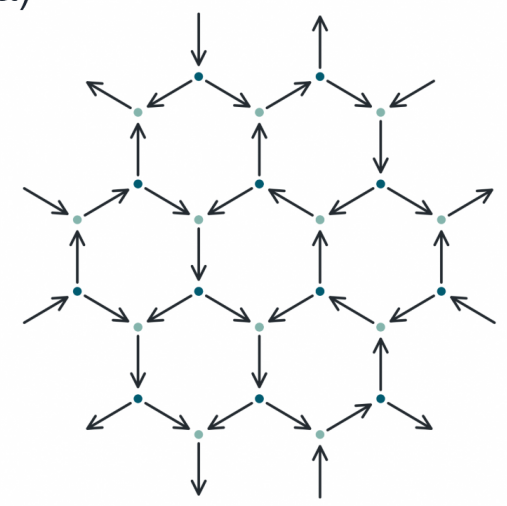

d)

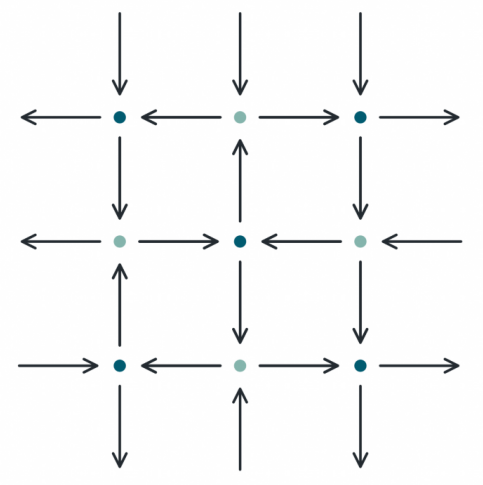

b)

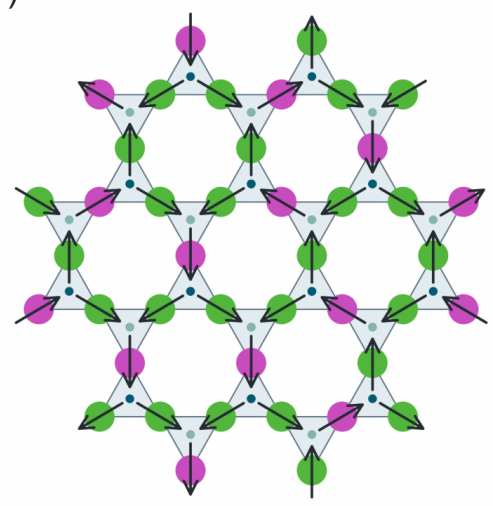

e)

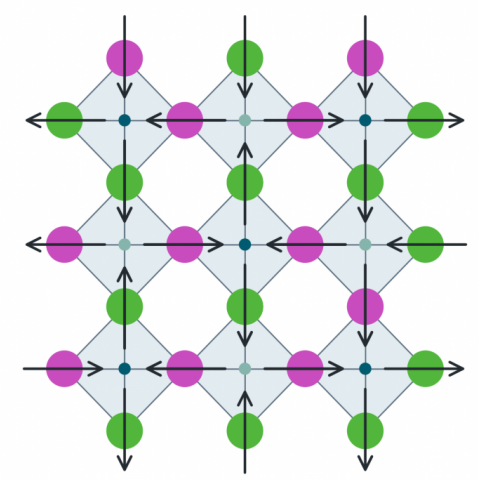

c)

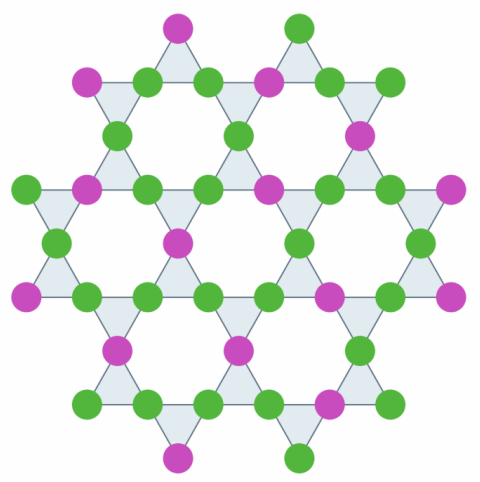

f)

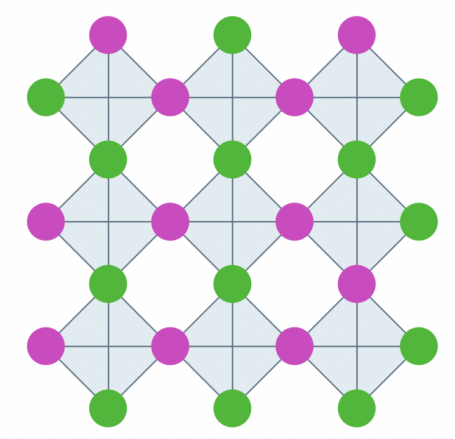

Figure S1: Equivalence of lattices and ice rules between the honeycomb (bond/spin ice) and kagome (site/spin-state ice) lattices (a-c); and equivalence of the square (bond/spin ice) and the putative checkerboard (site/spin-state ice) lattices (d-f). (a) In honeycomb artificial ice the spins (arrows in the figure) lie on the bonds of the lattice. Here we show a state that obeys the honeycomb 'ice II' rule: ${ }^{1}$ every site of one sub-lattice (marked by navy blue dots) has one spin pointing in and two spins pointing out; whereas every site on the other sublattice (pale blue dots) has two spins pointing in and one spin pointing out. (b) The same as (a), but we have placed a colored circle on each spin: purple if the arrow points from a pale blue site to a navy site; and green if the arrow points from a navy site to a pale blue site. The interactions between the spins are marked by blue lines. (c) Same as (b) but with the spins and blue dots removed. The kagome lattice structure is evident. If we now interpret purple circles as LS and green circles as HS the HSSI ice rule is obeyed - each blue shaded triangle contains two HS and two LS sites. (d) In square artificial ice the spins lie on the bonds of the lattice. Here we show a state that obeys the 2-in, 2-out ice rule: every site of the lattice (navy and pale blue dots, different shades indicate the two sublattices) has two spins pointing into it and two spins pointing out. (e) As (d), but with colored circles added on each spin: purple if the arrow points from a pale blue site to a navy site; and green if the arrow points from a navy site to a pale blue site. Interactions between sites are marked by blue lines. Diamonds with interactions across the diagonals are shaded blue. (f) As (e) but with the spins and blue dots removed. Interpreting purple (green) circles as LS (HS) a checkerboard spin-state ice rule is obeyed - every diamond shaded blue contains exactly two HS and two LS sites. 
S2 and S3.

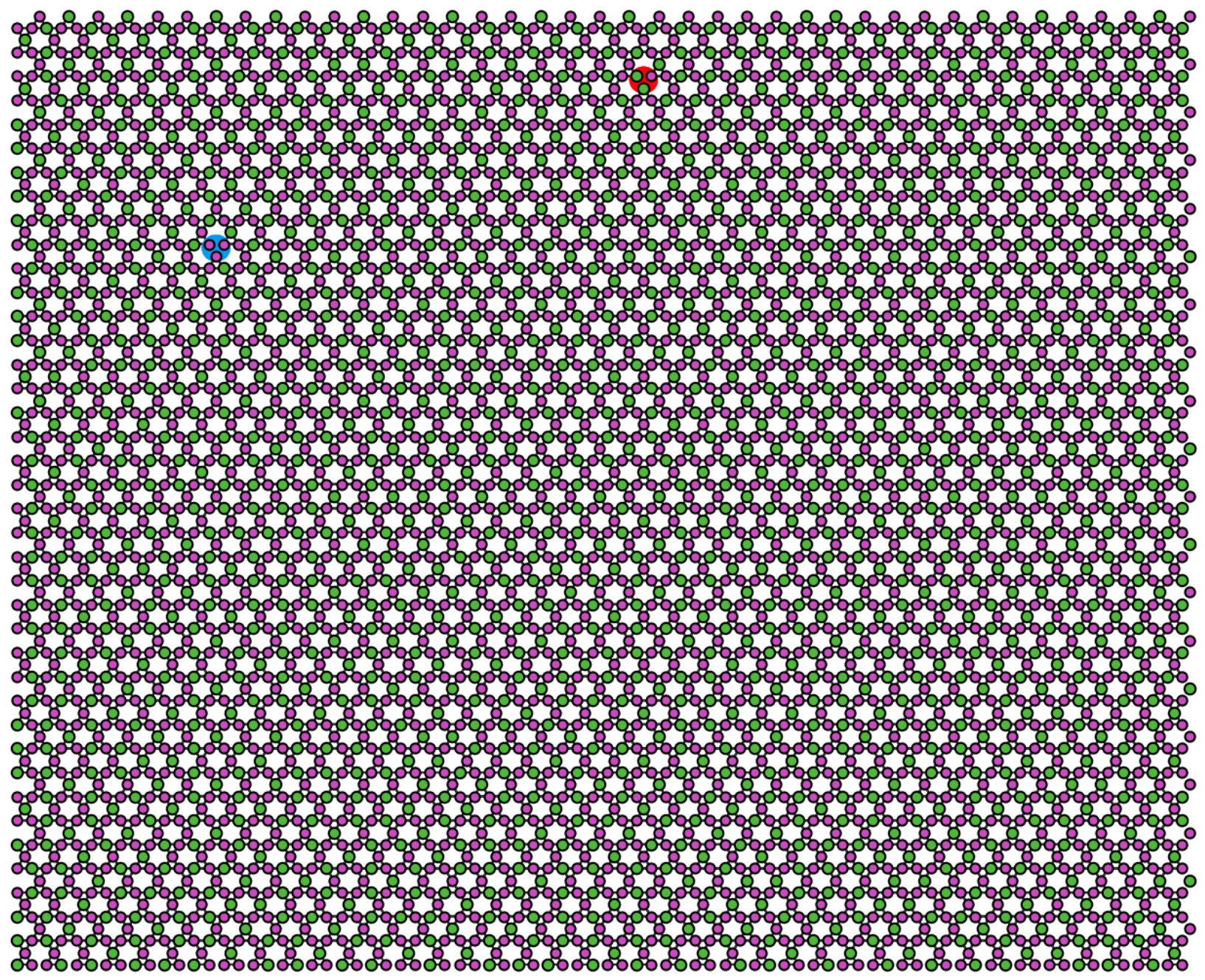

Figure S2: Snapshot of the full lattice from which Fig. 4a is taken.

\section{References}

(1) Nisoli, C.; Moessner, R.; Schiffer, P. Colloquium: Artificial spin ice: Designing and imaging magnetic frustration Rev. Mod. Phys. 2013, 85, 1473-1490. 


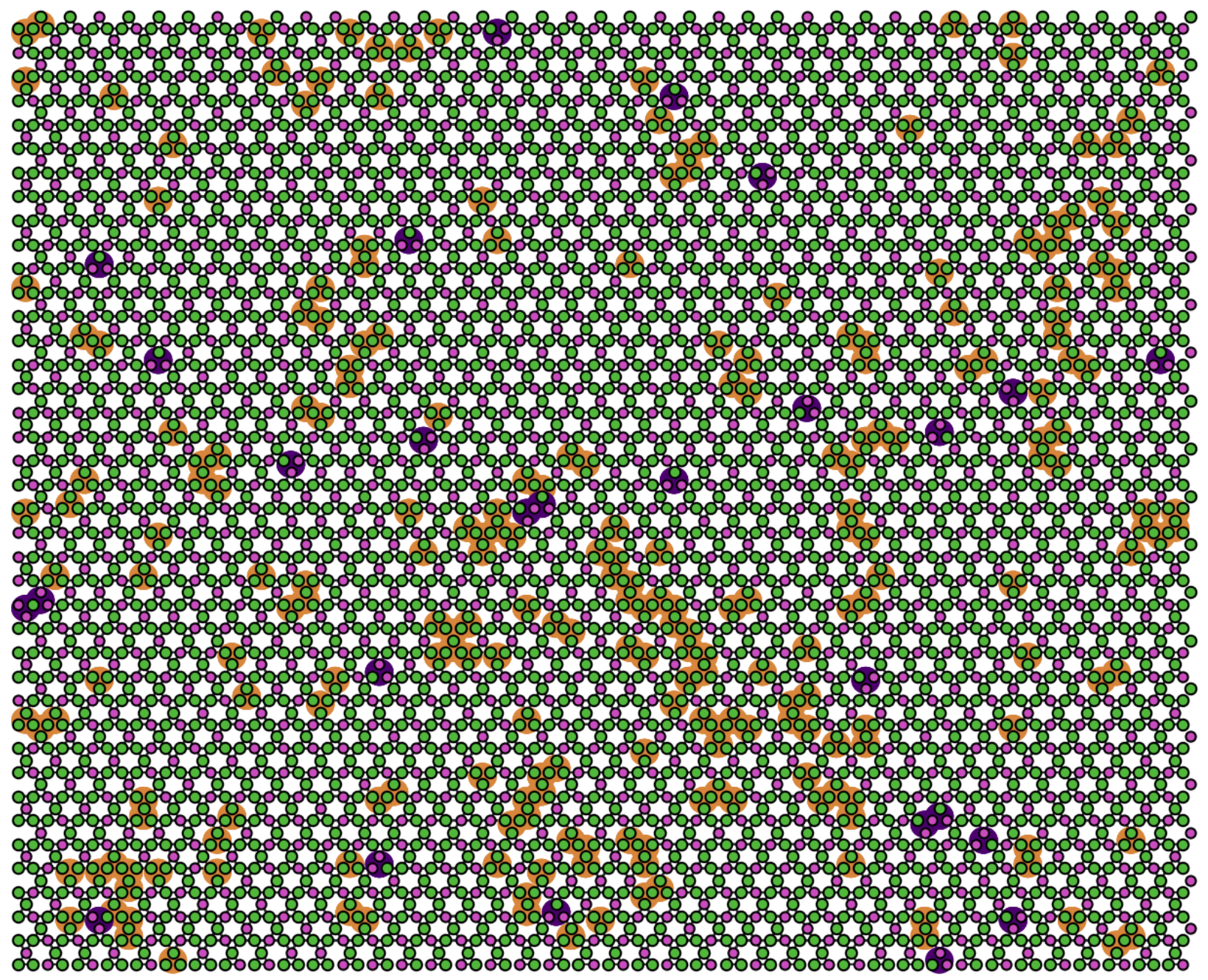

Figure S3: Snapshot of the full lattice from which Fig. 4b is taken. Note the tendency for the defects to cluster and that there are no triangles containing three LS metal centers, this is typical. 